\title{
ALBERTA
}

\section{Evaluation of Nursing Retention and Recruitment Programs}

\author{
Arlene Weidner, RN, MSc \\ Research to Action Project Coordinator \\ Calgary, $A B$ \\ Carol Graham, BA, LLB \\ Research to Action Project Coordinator \\ Calgary, $A B$ \\ Jennifer Smith, MA \\ President, Intergage Consulting Group \\ Ottawa, ON \\ Julia Aitken, BA \\ Intermediate Consultant and Researcher, Intergage Consulting Group \\ Ottawa, ON \\ Jill Odell, BA \\ Consultant and Researcher, Intergage Consulting Group \\ Ottawa, ON
}

\begin{abstract}
Retention and recruitment strategies are essential to address nursing workforce supply and ensure the viability of healthcare delivery in Canada. Knowledge transfer between experienced nurses and those new to the profession is also a focus for concern. The Multi-Employer/United Nurses of Alberta Joint Committee attempted to address these issues by introducing a number of retention and recruitment (R\&R) initiatives for nurses in Alberta: in total, seven different programs that were introduced to some 24,000 nurses and employers across the province of Alberta in 2001 (the Transitional Graduate Nurse Recruitment Program) and 2007 (the remaining six R\&R programs). Approximately 1,600 nurses participated in the seven programs
\end{abstract}


between 2001 and 2009. Of the seven strategies, one supported entry into the workplace, two were pre-retirement strategies and four involved flexible work options. This project entailed a retrospective evaluation of the seven programs and differed from the other Research to Action (RTA) projects because it was solely concerned with evaluation of pre-existing initiatives.

All seven programs were launched without a formal evaluation component, and the tracking of local uptake varied throughout the province. The union and various employers faced challenges in implementing these strategies in a timely fashion, as most were designed at the bargaining table during negotiations. As a result, systems, policy and procedural changes had to be developed to support their implementation after they became available.

Participants in the programs indicated improvements over time in several areas, including higher levels of satisfaction with work-life balance, hours worked and their current practice and profession. The evaluation found that participation led to perceived improvements in nurses' confidence, greater control over their work environment, decreased stress levels, increased energy and morale and perceived improved ability to provide high-quality care. However, no formal implementation plan had been developed or made available to assist employers with implementation of the programs. The findings highlight the need for more discipline in communicating, implementing and evaluating initiatives such as those evaluated retrospectively in this project. In particular, key performance indicators, baseline data, monitoring mechanisms and an evaluation plan need to be developed prior to implementation.

\section{Background}

In 2009, the Canadian Nurses Association report on the nursing shortage projected that unless immediate action was taken, Canada would be short 60,000 FTEs by 2022 (Tomblin Murphy et al. 2009). Sixty per cent of experienced nurses are over 40 years old, and healthcare administrators are anticipating a large number of retirements over the next few years (Coutts 2010).

Retention and recruitment strategies are an essential activity to address current and future supply issues in the nursing workforce and the viability of healthcare delivery in Canada. Growing demand, coupled with ongoing shortages, suggests that efforts must be increased to retain nurses currently working in the field. The 2008 national Nursing Turnover Study reported that the average turnover rate was close to $20 \%$ per year (27\% in ICU), costing an average of $\$ 25,000$ per nurse through temporary replacement costs and initial decreased productivity of new hires (O’Brien-Pallas et al. 2008). 
Further issues have arisen regarding the transfer of knowledge between experienced nurses and those new to the profession. Many feel that those entering the nursing workforce benefit from assistance in adjusting to the high stress and heavy workload. A 2004 study published in the Journal of Nursing Administration noted that the presence of experienced nurses in the workforce helps in the orderly transfer of knowledge to more recent graduates and inexperienced nurses (Duchscher and Cowin 2004). If greater numbers of experienced nurses contemplate retirement, health organizations will lose a tremendous source of knowledge and expertise.

There is now general agreement that a healthy work environment is an important component in any retention and recruitment strategy. A Journal of Advanced Nursing study on the determinants of hospital nurse intention to remain noted that "strategies that focus on building respectful relations at work may have tremendous capacity to promote nurse intention to remain employed" (Tourangeau et al. 2006). Unions, employers and professional associations believe there is a strong correlation between a healthy workplace and higher retention rates among experienced ( $>45$ years of age) nurses.

A 2006 Health Canada-funded study undertaken by the Canadian Federation of Nurses Unions (Wortsman and Janowitz 2006) reviewed recently published literature on retention of experienced nurses and conducted 30 structured telephone interviews with key informants representing unions, employers and government on current trends and nursing issues. In addition, input and insight into nurses' perceptions of their workplace environment and work life issues were obtained through a survey questionnaire distributed to 570 (285 returned) nurses and two focus groups. Findings from that study relevant to this project include the fact that many new graduates feel they are unprepared for the work environment and that too much is expected from them in the beginning. Better orientation and mentoring by experienced nurses was identified as key to assisting new graduates in their transition and integration into the workplace. The majority of nurses surveyed, 46 years of age and older, stated that changes in the work week, hours and flexible scheduling arrangements would greatly influence their decision to continue working rather than retiring. Strategies affecting retirement through phased-in retirement options or reduction of hours of work without reducing retirement benefits ranked first in overall desirability by nurses surveyed.

In response to increasing concerns over a nursing shortage, employers and the United Nurses of Alberta (UNA) introduced an ambitious list of retention and recruitment $(\mathrm{R} \& \mathrm{R})$ programs for nurses in Alberta. In total, there are seven 
initiatives available to approximately 24,000 nurses. The initial strategy, the Transitional Graduate Nurse Recruitment Program (TGNRP), was included in the 2001 Multi-Employer/UNA Collective Agreement and the additional six in the 2007 agreement.

Of the seven strategies, one supported entry into the workplace, two were preretirement strategies and four involved flexible work options

- To support entry into the workplace, the TGNRP, implemented in 2001, provided new graduates with supernumerary positions (positions above the normal staff complement) for up to one year.

- In 2007, the two pre-retirement strategies for nurses nearing retirement age were designed: under the Retirement Preparation Program, eligible nurses had $20 \%$ of their time to be designated for non patient-related work; and the Pre-Retirement FTE (full-time equivalent) Reduction Program reduced the FTE while maintaining the nurses' full pension.

- In addition, four flexible work options were designed for nurses at various stages in their careers. The first, the Weekend Worker Program, paid nurses working all weekends a full-time salary for 0.8 FTE of actual work. The second strategy, the Flexible Part-Time Program, allowed employees to increase their FTE hours while providing flexibility on scheduling additional shifts. The third strategy, the Seasonal Part-Time Position Program, was available to individuals who wished to compress their annual FTE into a smaller portion of the year and continue benefit coverage throughout the year. Finally, there was the Benefit-Eligible Casual Employee Position Program, which combined the flexibility of casual employment with a mutual commitment of a regular position with benefit coverage.

Approximately 1,600 nurses participated in the seven $R \& R$ programs between 2001 and 2009, with the majority (about 1,450) being Transitional Graduate Nurse Recruitment Program participants.

All seven programs were launched without a formal evaluation component, and the tracking of local uptake varied throughout the province. The focus of the Alberta Research to Action (RTA) initiative was to conduct a retrospective evaluation of the seven nursing $R \& R$ programs that were implemented. This RTA project differed from the other RTA projects because it was solely concerned with evaluation, not implementation, and was not part of the national evaluation by the Tomblin Murphy Consulting company. 


\section{Project Design and Implementation}

The project took place over a 27 -month period. A project steering committee was formed in fall 2008 and consisted of representatives from the UNA, Alberta Health Services (AHS), Covenant Health and Alberta Health and Wellness (AHW). Two project coordinators were contracted to manage the project. Intergage Consulting Group was engaged to complete the evaluation. The evaluation design received approval from the Conjoint Health Research Ethics Board (CHREB) at the University of Calgary. Throughout the project, an expert in social return on investment (SROI) and a videographer were contracted. The project required the assistance of key human resources contacts in the former health regions. These contacts were instrumental in providing the initial data for the preliminary mapping of the initiatives that had been implemented. Mapping included the historical health regions and identified individuals who had been involved with the implementation of the programs. The project coordinators worked with the College and Association of Registered Nurses of Alberta to provide a mailing list of Alberta nursing graduates from 2001 through 2009 to a contracted third-party mail-out service.

\section{Objectives}

The evaluation project set out to

- identify the sites where the seven retention and recruitment strategies had been implemented;

- evaluate the impact of the seven programs;

- determine, in particular, whether the retention and recruitment programs had a positive impact on quality of work life indicators; and

- contribute to the national body of evidence-based research on retention and recruitment initiatives.

\section{Methodology}

The evaluation methodology included a retrospective intentional design focus group with the steering committee, individual key stakeholder consultations and interviews, online surveys and site visits to five areas throughout the province. A total of 688 individuals participated in the various components of the evaluation.

Evaluation tools

\section{Consultations to confirm intentional design $(n=8)$}

A half-day consultation with the Alberta project steering committee was held to confirm a retrospective intentional design for the TGNRP and the various $\mathrm{R} \& \mathrm{R}$ programs. The discussion focused on two areas. The first was the design 
and implementation of the programs (rationale, objectives, use of supporting implementation plans, extent of consultation and engagement in the design and implementation, and challenges and opportunities related to design and implementation). The second was the success/impact of the programs (the committee's perception of the achievement of objectives, overview of key intended and unintended impacts and successes, the cost of not implementing, impact on partnerships and impact on sustainability and transferability).

\section{Mapping of programs and follow-up with selective sites}

Identification of participating organizations and facilities, and follow-up on preliminary data contained in a consolidated database, facilitated the mapping of the R\&R programs to support the evaluation. The data set for the evaluation surveys was generated from this mapping exercise.

\section{Key stakeholder interview consultations $(n=16)$}

A total of 16 key stakeholder interviews were conducted with selected participants, based on their experiences with the design and implementation of the $\mathrm{R} \& \mathrm{R}$ programs, their ability to provide feedback on how these were implemented and factors that may have supported or impeded success.

\section{Participant surveys $(\boldsymbol{n}=\mathbf{5 1 8})$}

The purpose of the two surveys (plus one follow-up) was to assess whether participating nurses were realizing expected outcomes, such as increased empowerment, job/professional satisfaction, impact on practice and occupational commitment (e.g., intention to stay in the profession) as a result of participation in these programs. Nurses were invited to participate in the two surveys via a mail-out letter. The surveys were hosted on the UNA website, and periodic monitoring was undertaken to gauge uptake and to follow up communications (by phone and e-mail, where available) to encourage participation.

- TGNRP Survey ( $\mathrm{n}=196 / 1,450) / N o n-T G N R P$ Survey ( $\mathrm{n}=234 / 3,555)$ : Given that participation in the TGNRP was not mandatory, a comparator group was identified for the TGNRP participants from nurses who had graduated in Alberta from 2001 to 2009, but had not participated in the TGNRP.

- R\&R Programs: Pre-Retirement and Flexible Work Options Initiative Participants Survey ( $\mathrm{n}=147 / 151)$ : While the initial methodology did not identify a comparator group in the six R\&R programs because of the small number of participants $(n=147)$, when the responses to the survey were analyzed, evaluators found that 54 of the individuals responding had not participated in any of the programs, thus serving as a comparator group for the six $R \& R$ 
programs. In addition, 22 individuals completed a follow-up survey eight months after completing the initial survey. The responses of these 22 individuals to the follow-up survey were compared directly to their original responses from the initial survey. R\&R program participation represents an actual response rate of $62 \%$. Thirty-six per cent of respondents who indicated they did not participate in any of these programs became the comparator group.

\section{Site visits (sites: $n=7$; implementers: $n=51$; nurses: $n=36$ )}

The site visit reviews included organizations where it was known that implementation of the R\&R programs had occurred in order to obtain first-hand information of effective implementation practices that may be transferable to other areas or professions. It also included organizations where participation in the $\mathrm{R} \& \mathrm{R}$ programs was lower than expected in order to identify potential barriers to implementation or suggested changes to the programs. Structured consultations were held with 51 implementers (managers, human resources, systems and other operations supports) and 36 nurses (who had either participated in one of the programs or who had indicated an interest in participating in them, and local UNA representatives).

\section{Limitations and Challenges}

The evaluation was retrospective in nature because the various programs had been implemented prior to approval of this evaluation project. There was very little quantitative baseline data (e.g., financial, overtime, sick time statistics) identified or gathered prior to or following implementation. There was also limited central tracking and monitoring of the programs that were implemented. As a result, it was difficult to obtain exact numbers or locations of implemented programs.

In addition, it is not known whether other projects or activities unrelated to the seven programs may also have influenced the survey indicators. Because of the lack of baseline data and monitoring of data elements throughout implementation, there was no ability to conduct statistical analysis on the data set that was ultimately compiled. It is acknowledged that there may be significant intervening variables that may have influenced the responses to the evaluation. These intervening factors may have included the major provincial restructuring of the Alberta health system with the merger of nine formerly separate health entities into one organization, Albert Health Services (AHS), in April 2009. As a result, the evaluation occurred within a dramatically changing healthcare environment in Alberta. The environment changed from one in which nurses were in great demand and initiatives to retain and recruit them were emphasized, to 
one in which nurses were perceived to be in excess supply and thus reduction in staffing levels was taking place. At the same time, the $\mathrm{H} 1 \mathrm{~N} 1$ pandemic was demanding significant attention and resources in most healthcare systems. This situation may have influenced the response rate from the nurses. It certainly contributed to the challenges of engaging AHS personnel, who were focused on both restructuring of the system and the pandemic response.

\section{Evaluation Findings}

\section{Program design}

According to all those consulted, at the time the TGNRP and six R\&R programs were introduced (2001 for the TGNRP and 2007 for the six R\&R programs), Alberta was experiencing a significant shortage of nurses. It was recognized that targeted actions were required in order to retain and recruit nurses and address nurses' and employers' needs. The UNA membership also wished to address workplace satisfaction. The TGNRP was introduced to assist with entry into practice, while the six $R \& R$ programs were introduced to provide more flexible work options (e.g., Weekend Worker, Flexible Part-Time, Seasonal PartTime and Benefit-Eligible Casual Employee positions). These were seen as a way to help stabilize the workforce and lead to greater satisfaction, and therefore greater retention among nursing staff. Further, the TGNRP was designed to assist other nurses with pre-retirement transition (e.g., the Retirement Preparation Program and the Pre-Retirement FTE Reduction Program).

During preparation for bargaining, the employer members of the Joint Committee noted that they developed a number of priority issues, criteria and principles for bargaining that included the need to ensure

- an adequate nursing supply and workforce;

- a high-quality workplace;

- safe patient care;

- a respectful environment in terms of transition and mentoring;

- effectiveness; and

- the addressing of strategic issues (e.g., retention of experienced RNs, responsiveness to environmental changes, appropriate use of health professionals, leadership and ensuring that the collective agreement wasn't acting as a barrier in the workplace).

In entering this round of negotiations, it was agreed that some creativity would be needed to design a series of initiatives that would effectively address the nursing shortage. This creativity was identified as the "predominant flavour 
throughout the bargaining process," with both parties bringing forward ideas for consideration and open discussion.

\section{Program implementation and supports}

Key informants and implementers consulted during the site visits noted that there was no formal plan developed or made available to assist employers with implementation of the programs. Implementers in all regions visited reported that only minimal supports were provided and that in most cases, implementation details were missing. This lack of detail led to delays in implementation, duplication of effort and inconsistent application throughout the province.

While key informants noted that the Joint Committee created guidelines for managers to implement the programs, employers had to develop policies and procedures according to who would or would not be accepted into a particular program. They also indicated that no adequate supports or guidance were put in place to assist in the required operational changes to payroll, scheduling, communication and management systems, or for the approval mechanisms required to implement the programs. All regions consulted felt that they were left to decide whether and how to implement the R\&R programs. As a result, the initiatives were applied differently in each region, increasing the difficulty of supporting, monitoring and evaluating them at the provincial level.

\section{Transitional Graduate Nurse Recruitment Program (TGNRP)}

Overall, the program had a positive impact on nurses' confidence, on their skills and ability to provide high-quality patient care and work collaboratively with other team members, and on their job satisfaction. The most valued aspects identified were the mentoring relationship, learning opportunities and the time allowed for them to learn their new role. Consultations through the site visits indicated a high level of satisfaction with the TGNRP, both among managers and TGNRP participants:

- Forty-three per cent of respondents identified that their motivation for participating in the TGNRP was to gain knowledge and experience; $27 \%$ responded that their motivation was to ease their transition into practice.

- Fifty-six per cent responded that the aspects they liked most about the TGNRP were the support, guidance and mentoring they received (Figure 1).

- The large majority (84\%) indicated that they would participate in the program again. This finding would indicate that TGNRP respondents found value in the program. 


\section{Figure 1.}

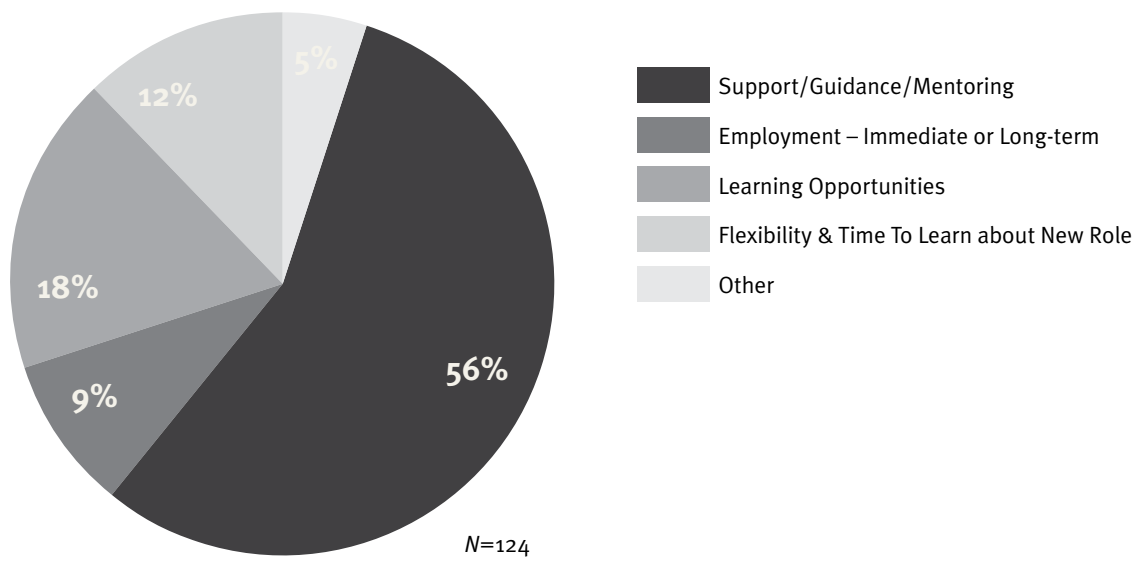

- Although a maximum of one year was identified in the program description, it was noted by $77 \%$ of participating nurses that six to nine months was adequate. In addition, this time period provided new graduates with adequate opportunity to apply for regular full-time positions in their practice area of interest. It was hoped that employers would be able to retain these nurses in their organizations for a longer period, assuming that the nurses would have greater satisfaction with their workplace and practice area.

- The key attribute identified both by participating nurses and by implementers was the need to allow flexibility based on the individual participant's own needs and the individual's speed of development in the program. There was, however, a lack of standardization in the length of the program, the training materials available and the provision of a consistent mentor. Improved guidelines and clearly articulated expectations may help to achieve outcomes more effectively.

- Of TGNRP participants who responded $(n=82)$ to open-ended questions about those aspects of the program they did not like, 15\% noted having "no mentor," $12 \%$ indicated "insufficient staff support/buy-in/little support from unit supervisors and senior nurses for program," 10\% indicated "limited time with mentor" and 9\% identified "multiple mentors" as major reasons for not liking the program (Figure 2). These findings reflect the inconsistency with which the program was implemented across sites, some of which may be attributed to the challenges and confusion noted with regard to implementation. 
- When asked how they would rate the TGNRP overall, most (67\%) of the respondents who answered this question rated the program as excellent/very good. A further $21 \%$ rated it as good and $8 \%$ of respondents rated it as poor (Figure 3 ).

\section{Figure 2.}

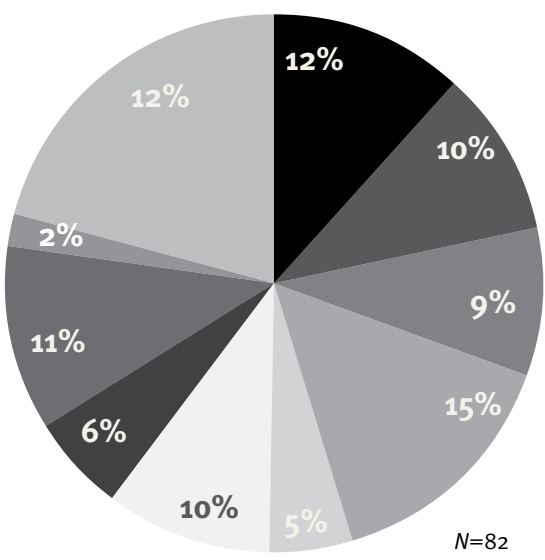

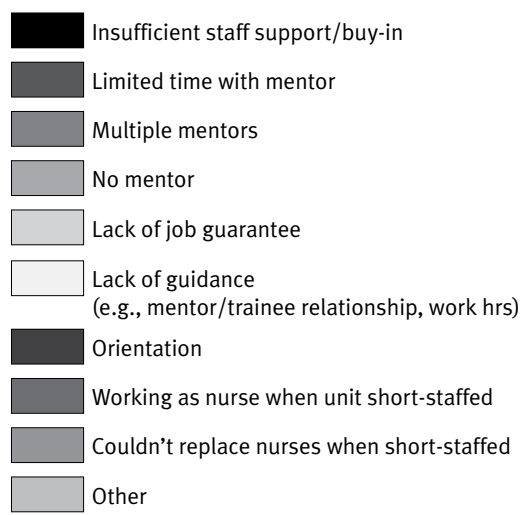

\section{Figure 3.}

Overall rating of TGNRP by participants

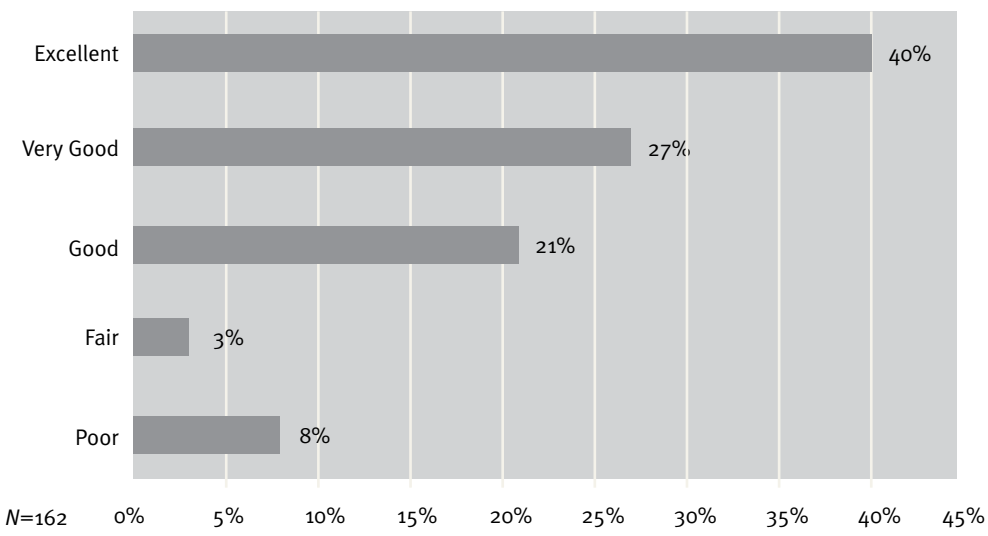


Respondents were asked, in an open-ended question, what they felt could be done to improve the TGNRP (Figure 4). Useful suggestions included:

- Ensure staff buy-in/resource allocation so that staff in the work unit understand what the program is for, its scope and importance. Also, ensure that participants are assigned mentors with sufficient time in their schedules to perform their mentoring duties.

- Standardize the program to include training materials and guidelines for trainees and mentors that give clear expectations for the work unit.

- Standardize learning materials, orientation programs and online resources for nurses to access outside their clinical work hours.

- Continue/expand the program to make it mandatory for all new nurses and to ensure its availability to everyone, i.e., increase access (equivalent to a nursing internship).

- With regard to length and timing, the program should be at least six months in length, and the start dates should be staggered so as not to overwhelm units with too many new graduates.

- Share mentoring responsibilities among a team within the unit.

- Guaranteed employment would be a great incentive. Not having this guarantee seems a waste of resources, given the significant investment in the TGNRP year, if no positions are available for individuals to move into upon completion of the program.

\section{Figure 4.}

"How might the TGNRP be improved in the future?" (TGNRP respondents)

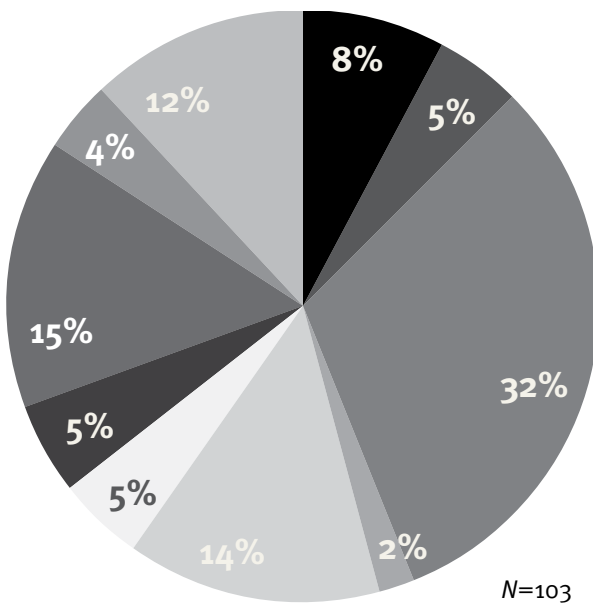

\begin{tabular}{|l}
\hline Continue/Expand \\
Length/Timing \\
Staff/Buy-in/Resource Allocation \\
More independence \\
Learning Materials \\
Team Mentoring \\
Provide Employment Afterward \\
Standardization \\
Protecting Trainees as Trainees \\
Other
\end{tabular}


TGNRP respondents were generally more positive than non-TGNRP respondents about their future plans as nurses, indicating a desire to stay longer in their current position and in the nursing profession (Figure 5):

- Sixty per cent of non-TGNRP respondents strongly agreed/agreed that they planned to stay in the same clinical site over the next year, compared to $72 \%$ of TGNRP respondents.

- Approximately $80 \%$ of TGNRP respondents strongly agreed/agreed that it was unlikely they would leave the profession in the next year. This percentage was similarly found among non-TGNRP respondents.

- A slightly higher percentage of non-TGNRP respondents agreed that they frequently think about leaving the profession (21\% vs. $15 \%)$ and regretted their choice to enter the nursing profession (15\% vs. 10\%).

\section{Figure 5.}

Views of nurses participating in the TGNRP $(n=167$;

${ }^{\left.*_{n}=166\right)}$ vs. those of comparator nurses $(n=223)$

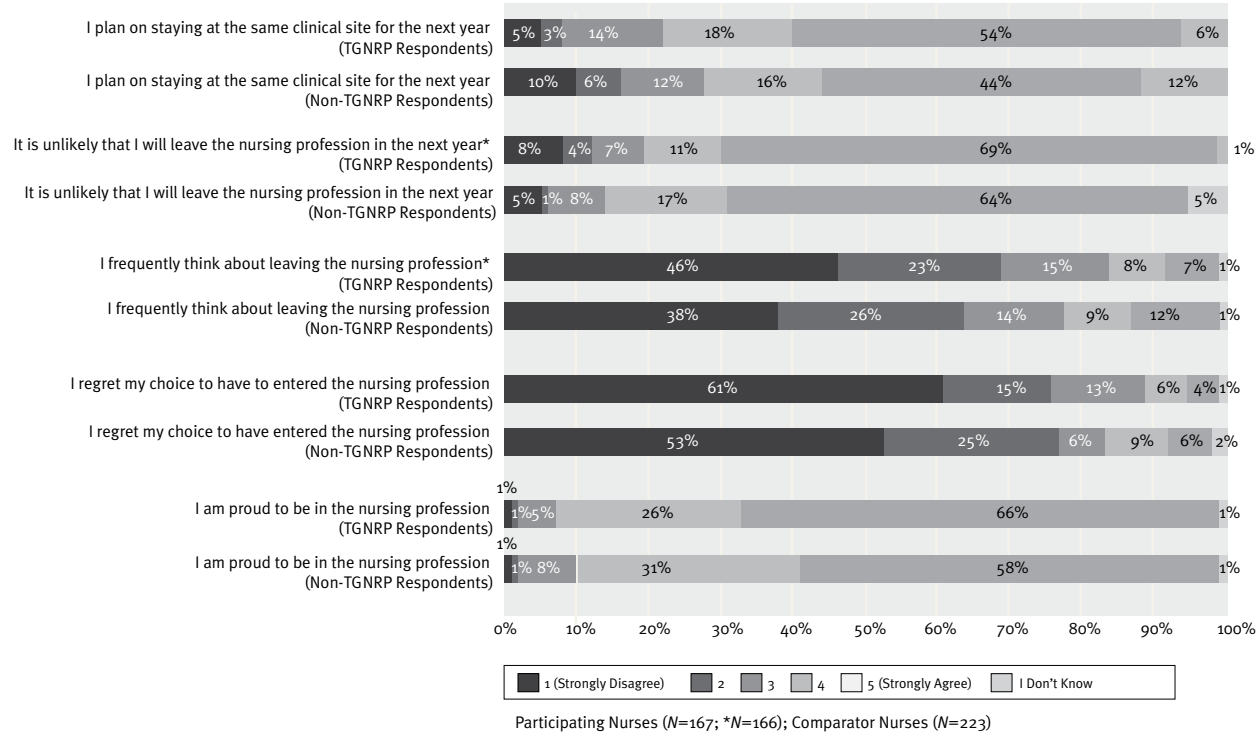

\section{Other six R\&R programs}

This component of the R\&R program survey attempted to obtain participating nurses' feedback about their level of satisfaction prior to participation in their respective program. Generally, very few nurses were satisfied with the balance 
between work and personal life prior to engaging in their respective program. Interestingly, nurses in the Seasonal Part-Time positions seemed to indicate the highest level of satisfaction with their quality of work-life balance prior to starting the program.

Benefit-Eligible Casual Employee Program: This was deemed an important retention tool, as it allowed managers flexibility in offering alternatives to a casual position when there were no opportunities for full-time and regular part-time work. Participants reported greater job security, access to benefits and improved control over their work-life balance. Key challenges included making adjustments to payroll systems and managing expectation when demand exceeded the number of available positions.

Weekend Workers: This program was intended to make weekend shifts more attractive and easier to staff. It was also designed to support improved work-life balance. One of the key challenges was the cost and perception of paying nurses a full-time salary for 0.8 FTE of actual work. Participation in this program had a positive impact on job satisfaction and quality of care provided and allowed managers more time to focus on patient care instead of scheduling.

These positions, however, were sometimes implemented in areas where there may not have been an operational need but rather a desire on behalf of the nurses to access these jobs. In those instances, some nurses who were interested in weekend work resented those who were in the program. Challenges also arose with senior individuals in these positions taking their vacation, requiring a significant number of weekend shifts needing to be filled. The program also required payroll system changes that sometimes had to be done manually.

Pre-Retirement FTE Reduction: This program involved limiting the workload of those nearing retirement by reducing their FTE by up to 0.2 for no more than 2.5 years prior to leaving the workforce. Participants stated they had more time to re-energize and so they felt less drained during the hours they worked and were able to provide better patient care. Participants also identified greater job stability. Employers, however, were challenged to fill the remaining 0.2 FTE after participants had their workloads reduced by that amount. As well, there was no clear direction on how to revert the position back to the previous FTE after the participant retired.

Retirement Preparation: To assist those nearing retirement, this program focused on allocating nurses' FTE between patient care and work associated with projects, 
proposals and mentoring, with $20 \%$ of their time designated for non-patientrelated work. In some cases, however, it was difficult to determine the research proposals and projects that the employees were to work on during the designated $20 \%$ of their time. Often, participants felt pressured to cover staffing shortages.

Flexible Part-Time: The purpose of this program was to allow employees to increase their FTE while providing flexibility on the scheduling of additional shifts. Only one participant commented on her involvement with this position. It is unclear whether the limited uptake was due to a lack of interest, awareness or communication about the program. A key challenge for managers aiming to provide flexible scheduling for part-timers would be to coordinate the schedules of nurses selecting this option with the schedules of regular full-time staff.

Seasonal Part-Time: This option was available to those individuals who wished to work for a designated amount of time (e.g., six months with the remaining six months off; useful for full-time students or those wishing not to work for either the winter or the summer). Although the aim of this program was to improve work-life balance, certain participants (this option included four participants) noted they did not experience a significant change in the level of work still required. In addition, managers found it difficult to find someone who was interested in working the remaining six months, and also identified the need for re-orientation required for the staff upon their return. Of the participants that enrolled in seasonal part-time positions, there was a high level of satisfaction with hours worked and leadership within their units. Those consulted indicated improved work-life balance.

It is clear that involvement in the R\&R programs had an impact on the level of satisfaction among participants. Based on the results from the R\&R programs follow-up survey, the greatest impact was shown in the following areas of practice:

- Hours of work: 91\% either agreed or strongly agreed that their level of satisfaction with their current job was influenced by participation in their $R \& R$ program. This is not surprising, as most of the programs resulted in the nurses' working fewer hours, having more flexible schedules or having more control over their work hours.

- Balance between work and family/personal life: 91\% agreed or strongly agreed that their level of satisfaction with their current job was influenced by participation in their R\&R program.

- Current job: 78\% either agreed or strongly agreed that their level of satisfaction with their current job was influenced by participation in their $R \& R$ 
program, with 14\% strongly disagreeing.

- Nursing profession: 68\% either agreed or strongly agreed that their level of satisfaction with the nursing profession was influenced by participation in their R\&R program, with 19\% disagreeing and another 14\% neutral.

- Practice setting/work environment: $68 \%$ agreed or strongly agreed that their level of satisfaction with their current job was influenced by participation in their R\&R program, with 19\% disagreeing and another 9\% neutral.

Less than $50 \%$ of respondents agreed that their level of satisfaction with respect to areas of practice, such as "the nursing leadership in my unit" and the "level of collaboration among healthcare providers in my unit," was affected by program participation.

Figure 6 displays the post-survey results regarding the broader impacts of program participation. The impact of the $R \& R$ programs is primarily related to increased satisfaction with current job and improved work-life balance.

\section{Figure 6.} Impact of participation in the R\&R programs

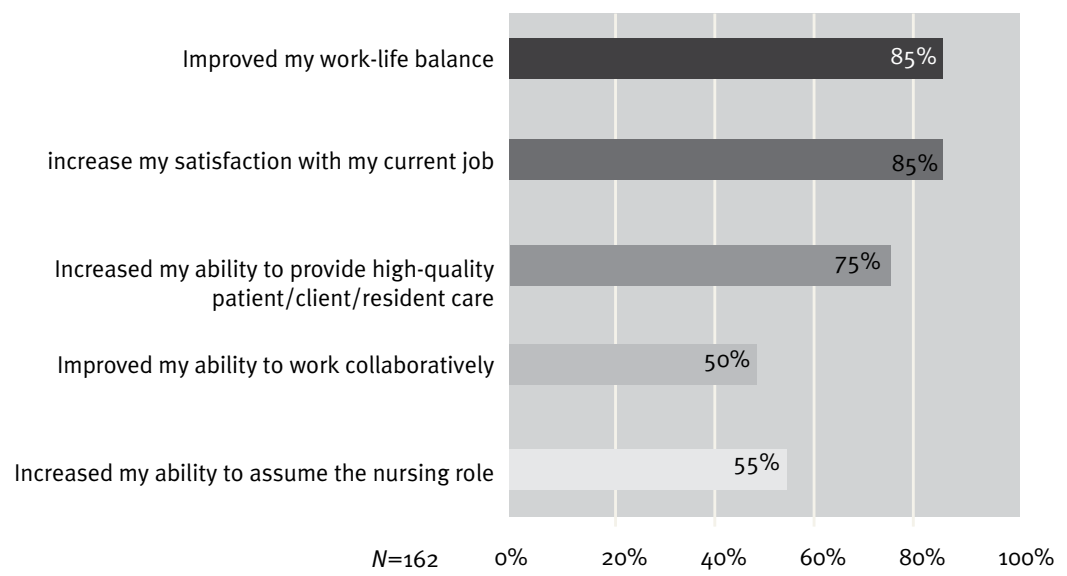

An overwhelming majority of respondents assessed the $\mathrm{R} \& \mathrm{R}$ programs as either excellent or very good (65\% and $20 \%$, respectively); $95 \%$ rated them from good to excellent. The remaining $5 \%$ of individuals rated the R\&R programs fair (Figure 7). 


\section{Figure 7.}

"Overall, how would you rate the R\&R program?" (Follow-up survey respondents)

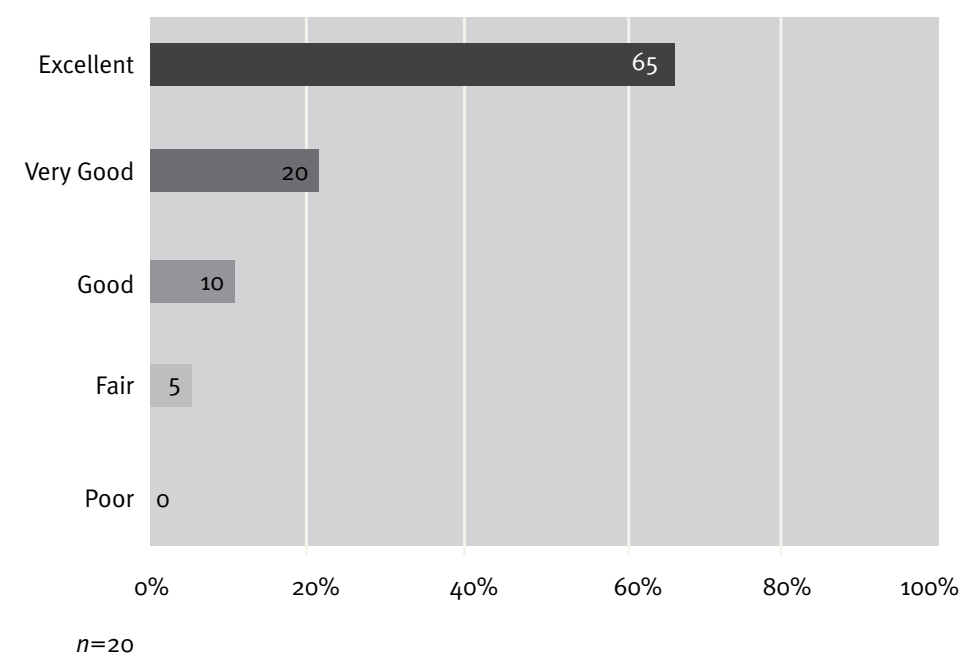

\section{Lessons Learned}

The evaluation revealed that stakeholders viewed all the R\&R programs (particularly the TGNRP, Weekend Worker, BECE and Pre-Retirement FTE Reduction programs) as having a positive impact on the retention and recruitment of nurses. The programs were viewed as helping to address specific workforce needs as well as the needs of employees. The majority of key informants agreed that these initiatives should and could be transferred to other health facilities and health professions (indeed, any shift worker) based on the required need.

The RTA project findings highlight the need for more discipline in communicating, implementing and evaluating initiatives such as those that were evaluated retrospectively in this project. In particular, key performance indicators, baseline data, monitoring mechanisms and an evaluation plan need to be developed prior to implementation. More could have been done to increase awareness among key stakeholders (managers, nurses, union representatives and other healthcare providers) about the seven programs. Knowing the operational details for the implementation of any initiative is important in order to understand any payroll system challenges, ensure provincial consistency and avoid duplication of effort. As well, the state of the local labour-management relationship was an important factor in supporting successful implementation of the programs. 
The findings of the evaluation were presented to the Multi-Employer/UNA Joint Committee. The following activities were highlighted as key to improving the planning and implementation of current and future retention and recruitment initiatives:

- Need for a broad engagement of stakeholders to inform the design and implementation of future initiatives.

- Detailed broad-scale implementation plan to support a provincial roll-out with a focus on the longer term (three years and more); a toolkit to support employers and managers with a greater consistency of implementation.

- Identification of key performance indicators to support monitoring strategies.

- Communication strategy to ensure all stakeholders are aware of the initiatives and of a recommended approach to implementation.

- Evaluation plan to be in place before implementation, which includes a social return on investment (SROI) element.

The Joint Committee accepted the recommendations and is developing a plan to inform decision-making and implementation of future collective bargaining and Joint Committee initiatives.

\section{References}

Coutts, J. 2010. Experts and Evidence: Opportunities for Nursing. Ottawa: Canadian Federation of Nurses Unions. Retrieved November 13, 2011. <http://www.nursesunions.ca/report-study/ experts-evidence-opportunities-in-nursing $>$.

Duchscher, J.E.B. and L. Cowin. 2004. "Multigenerational Nurses in the Workplace." Journal of Nursing Administration 34(11): 493-501.

O’Brien-Pallas, L., G. Tomblin Murphy and J. Shamian. 2008. Understanding the Costs and Outcomes of Nurses' Turnover in Canadian Hospitals. Nursing Turnover Study FRN\#66350. Toronto: Nursing Health Services Research Unit, University of Toronto.

Tomblin Murphy, G., S. Birch, R. Alder, A. MacKenzie, L. Lethbridge, L. Little and A. Cook. 2009. Tested Solutions for Eliminating Canada's Registered Nurse Shortage. Ottawa: Canadian Nurses Association.

Tourangeau, A.E., G. Cummings, L.A. Cranley, E.M. Ferron and S. Harvey. 2006. “Determinants of Hospital Nurse Intention to Remain Employed: Broadening Our Understanding." Journal of Advanced Nursing 66(1): 22-32.

Wortsman, A. and S. Janowitz. 2006. Taking Steps Forward: Retaining and Valuing Experienced Nurses. Ottawa: Canadian Federation of Nurses Unions. Retrieved November 13, 2011. <http:// www.nursesunions.ca/report-study/report-taking-steps-forward-retaining-and-valuing-experienced-nurses-january-26-2006>. 